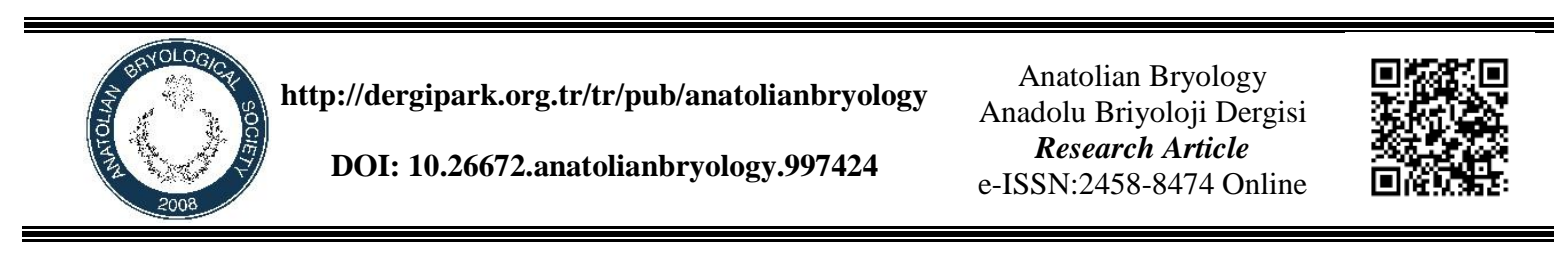

\title{
Bryodiversity of Eflani District of Karabük Province
}

\author{
Ahmet ARSLAN $^{1}$ (D) , Ayșe Dilek UNAN ${ }^{1}$ (D), Muhammet ÖREN ${ }^{1 *}$ \\ ${ }^{1}$ Zonguldak Bülent Ecevit University, Faculty of Science and Art, Department of Biology, Zonguldak. \\ TURKEY
}

\begin{abstract}
\begin{tabular}{lll}
\hline Received: 19 September 2021 & Revised: 02 October 2021 & Accepted: 13 October 2021
\end{tabular}
Abstract

Eflani district of the Karabük province selected as the study area is located Western Black Sea Region. Three field excursions have been made in different seasons between October 2017 to April 2019 in the study area. Bryophyte specimens were collected from 30 different habitats and ecosystems such as steppes, forests, riparian, farmlands, wetlands etc. vegetation. Approximately 500 specimens have been collected, examined under stereo and light microscopes, and identified using related literature. 156 specific and infraspecific bryophyte taxa (15 liverworts and 141 mosses) were found in the visited localities. Among them, 21 taxa were reported from Karabük province for the first time and Lewinskya acuminata (H.Philib.) F.Lara, Garilleti \& Goffinet was new for A2 grid square. These taxa were listed according to taxonomic hierarchy with locality, habitat, and substrate information.
\end{abstract}

Key words: Bryophyte, flora, Eflani, Karabük, Turkey

\section{Karabük İli, Eflani İlçesi’nin Briyofit Çeşitliliği}

Öz

Çalışma alanı olarak seçilen Karabük İli, Eflani İlçesi, Batı Karadeniz Bölgesi sınırları içerisinde yer almaktadır. Çalışma alanına Ekim 2017-Nisan 2019 tarihleri arasında farklı sezonlarda 3 arazi gezisi düzenlenmiştir. Briyofit örnekleri; step, orman, dere kenarları, sulak alanlar, tarım arazileri gibi. farklı habitat ve ekosistemleri içeren 30 farklı lokaliteden toplanmıştır. Toplanan yaklaşık 500 örnek, 1şık ve stereo mikroskop altında ilgili yayınlar kullanılarak teşhis edilmiştir. Ziyaret edilen lokalitelerden tür ve türaltı kategoride 156 briyofit taksonu (15 ciğerotu ve 141 karayosunu) tespit edilmiştir. Bunlardan 21 tanesi Karabük ilinden ilk defa bildirilirken, Lewinskya acuminata (H.Philib.) F.Lara, Garilleti \& Goffinet türü A2 karesinden ilk kez kaydedilmiştir. Bulunan taksonlar sistemik hiyerarşiye uygun olarak lokalite, habitat ve substrat bilgileri ile birlikte listelenmiştir.

Anahtar kelimeler: Briyofit, flora, Eflani, Karabük, Türkiye.

\section{Introduction:}

Bryophytes are a primitive and non-tracheophyte group of land plants. They are dominant plants of some ecosystems and have a worldwide distribution, from the equator to the poles. This group includes 20.000-25.000 species and a very important role in their ecosystems (Glime, 2021).

\footnotetext{
* Corresponding author: muhammetoren@ hotmail.com

(C) 2021 All rights reserved / Tüm haklarl saklıdır.

To cite this article: Arslan A. Ünan A.D. Ören M. 2021. Bryodiversity of Eflani District of Karabük Province. Anatolian Bryology. 7:2, 146-155.

(c) (1) \& This work is licensed under a Creative Commons Attribution-Non Commercial 4.0 International License.
} 
Turkey inhabits approximately 1000 bryophyte species. This number indicates that Turkey has the highest biodiversity among the Southwest Asian countries (Kürschner and Frey, 2020). Because of different habitat types, geology, soil types, topography, climates, and also its biogeographic location, Turkey has the potential of hosting a vast amount of bryophyte species with different ecological requirements. Thus, many new bryophyte records, have been reported the different localities of northern part of the country, in recent years (Gözcü et al., 2019; Özenoğlu et al., 2019; Ursavaş and Keçeli, 2019; Ursavaş and Işın, 2019; Erata and Batan, 2020; Abay et al., 2021; Erata et al., 2021; Unan and Ören, 2021; Unan et al., 2021; Ursavaş et al., 2021). It is seen that even from Northern Turkey, where many bryofloristical studies have been carried out, new records are still being given. These areas are characterized by oceanic or rainy Mediterranean climate and related to bryologist researchers' workplace. From this point of view, in order to achieve a better understanding of the bryofloristic structure of Turkey, floristically unstudied parts of the country with different ecosystems and climate conditions should be promptly investigated in detail.

\section{The Study Area}

The study area, Eflani is a district of the Karabük province located in the Northern West Black Sea region (Fig. 1). The district covers an area of 536 $\mathrm{km}^{2}$, and the altitude is $910 \mathrm{~m}$ a.s.l. at the city center. The population density of Eflani is fairly low, the population of the city center is approximately 2240 , while the total population is 9700 including villages (URL 1).

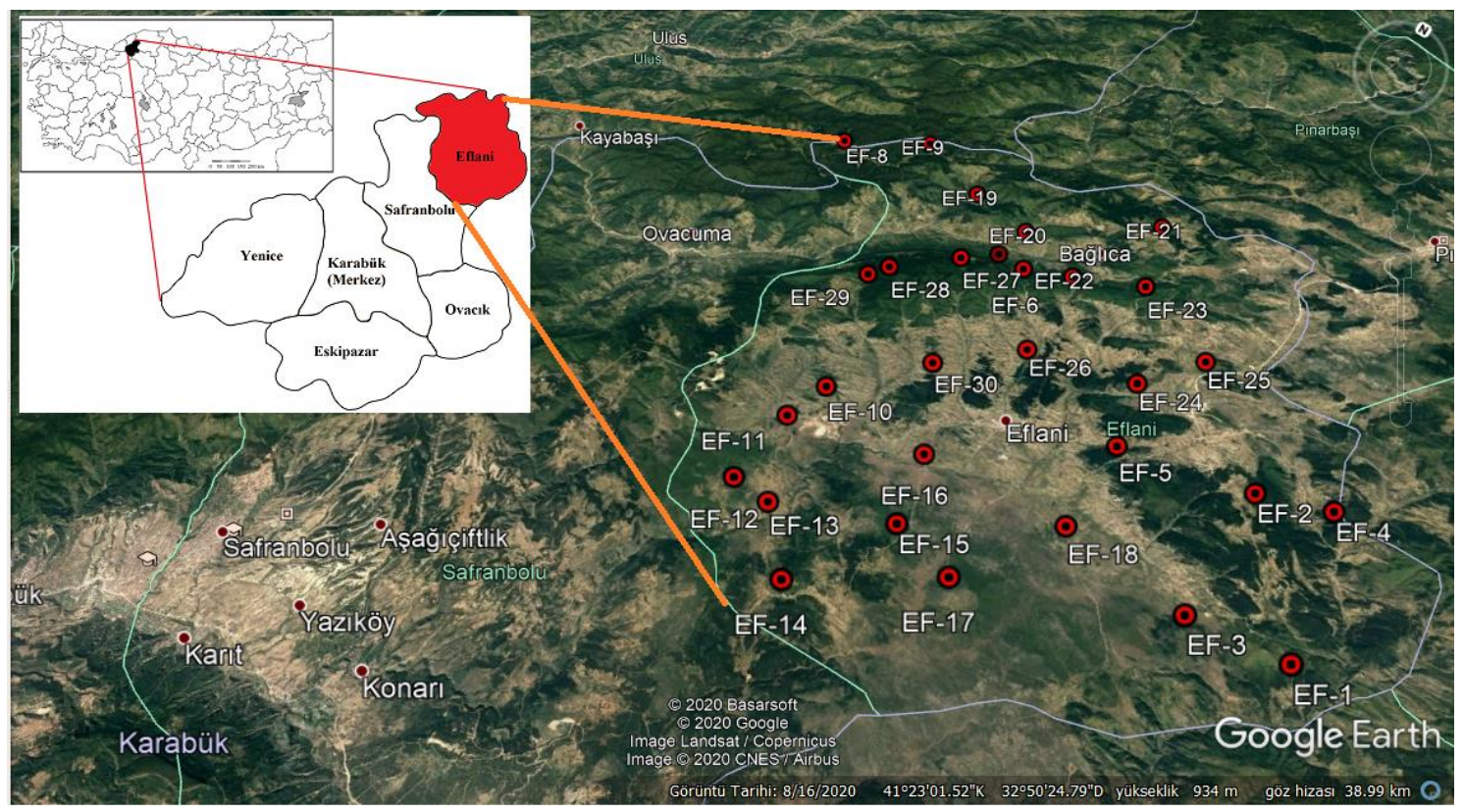

Fig. 1. The map of the Eflani district and collecting points.

The study area is located in the climatic transition zone: northern parts of the district are cool and rainy, and southern parts are relatively hot and dry. According to Köppen-Geiger classification, Eflani climate has $\mathrm{Cfb}$ type climate. This climate type is characterized by warm winters and summers, and precipitation throughout the year. The annual average temperature is around $9.9{ }^{\circ} \mathrm{C}$, and the annual average precipitation is around $716 \mathrm{~mm}$ (Arslan, 2021). Considering the distribution of precipitation according to the seasons, it is listed as $223 \mathrm{~mm}$ in winter, $205 \mathrm{~mm}$ in spring, $149 \mathrm{~mm}$ in autumn and $139 \mathrm{~mm}$ in summer, and the precipitation regime is Eastern Mediterranean 1st type, KIYS, (Winter, Spring, Summer, Fall) (Akman, 1999).
Eflani has different ecosystems and habitats. Approximately $40 \%$ of the district consists of agricultural areas, $43 \%$ forests, $6 \%$ meadows and pastures, $10 \%$ settlements and other areas (steppe, rocky areas etc.) (URL 2). Pinus nigra subsp. pallasiana (Lamb.) Holmboe, Abies nordmanniana subsp. equi-trojani (Asch. \& Sint. ex Boiss.) Coode \& Cullen, Quercus sp. L., Carpinus betulus L., Fagus orientalis Lipsky, Pinus sylvestris L., Juniperus sp. L. are dominant trees in the forests. The other woody plants are Alnus glutinosa (L.) Gaertn., Corylus avellana L., Carpinus betulus L., Cornus mas L., Pyracantha coccinea M.Roem., Populus tremula L., P.nigra L., Salix sp. L., Crataegus sp. L.. 
The main rocks types are respectively clastic, clastic and carbonate, neritic limestone, carbonate and clastic (dated back to Middle Devonian - Lower Carboniferous), carbonate and clastic rocks (dated back to Ordovician - Lower Devonian) (URL 3). Brown soils cover a large area in and around Eflani. It is also possible to find alluvial soils around the rivers. Other soil types are colluvial, gray-brown podzolic, brown forest, non-calcareous brown forest soils in the research area (URL 1).

\section{Material and Methods}

In order to reveal the bryophyte flora of Eflani district of Karabük province, plant specimens were collected from 30 points by visiting the area between October 2017 and April 2019 at different seasons of the year. In the selection of sampling points, attention was paid to include different elevations, habitats, forest types and coordinates that could represent the whole area.

\section{Collecting localities}

1- Karabük, Eflani, between Başiğdir-Demirli village, $41^{\circ} 22^{\prime} 19.2^{\prime \prime} \mathrm{N} \quad 033^{\circ} 06^{\prime} 33.1^{\prime \prime E}$, Pinus nigra subsp. pallasiana (Lamb.) Holmboe, Carpinus betulus L., Quercus sp. L., Juniperus sp. L., 1092 m, 14.10.2017.

2- Karabük, Eflani, Abakolu Village,

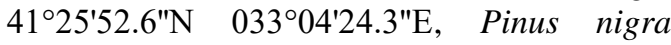
subsp. pallasiana (Lamb.) Holmboe, Quercus sp. L., 1140 m, 14.10.2017.

3- Karabük, Eflani, Abakolu Village, Örencik vicinity, $41^{\circ} 22^{\prime} 19.2^{\prime \prime} \mathrm{N} \quad 033^{\circ} 03^{\prime} 54.7^{\prime \prime} \mathrm{E}$, open lands, $1088 \mathrm{~m}, 14.10 .2017$.

4- Karabük, Eflani, Karlı Village, 41ํำ'15.1"N 03306'23.0"E, Pinus nigra subsp. pallasiana (Lamb.) Holmboe, P. sylvestris L., $989 \mathrm{~m}$, 14.10.2017.

5- Karabük, Eflani, Urban Forest, around Ortakçiler Pond, $41^{\circ} 25^{\prime} 44.7^{\prime \prime} \mathrm{N} 033^{\circ} 00^{\prime} 39.0^{\prime \prime} \mathrm{E}$, Pinus nigra subsp. pallasiana (Lamb.) Holmboe, Pinus sylvestris L., $935 \mathrm{~m}$, 14.10.2017.

6- Karabük, Eflani, Seferler Village vicinity, $41^{\circ} 29^{\prime} 55.3^{\prime \prime N} 032^{\circ} 55^{\prime} 41.5^{\prime \prime E}$, Populus tremula L., Quercus sp. L., Carpinus betulus L., Fagus orientalis Lipsky, Abies nordmanniana subsp. equi-trojani (Asch. \& Sint. ex Boiss.) Coode \& Cullen, 1053 m, 14.10.2017.

7- Karabük, Eflani, Seferler Village vicinity, $41^{\circ} 30^{\prime} 09.0^{\prime \prime} \mathrm{N} 032^{\circ} 54^{\prime} 44.3^{\prime \prime E}$, Fagus orientalis Lipsky, Abies nordmanniana subsp. equitrojani (Asch. \& Sint. ex Boiss.) Coode \& Cullen, 944 m, 14.10.2017.

8- Karabük, Eflani, Ovaşeyhler Village vicinity, $41^{\circ} 32^{\prime} 20.1^{\prime \prime} \mathrm{N} \quad 032^{\circ} 47^{\prime} 45.0^{\prime \prime} \mathrm{E}, \quad$ Abies nordmanniana subsp. equi-trojani (Asch. \&
Sint. ex Boiss.) Coode \& Cullen, Salix sp. L., $956 \mathrm{~m}, 15.10 .2017$.

9- Karabük, Eflani, Akçakese Village, Bostancı vicinity, $41^{\circ} 33^{\prime} 19.8^{\prime \prime} \mathrm{N}$ 032॰50'25.7"E, Abies nordmanniana subsp. equi-trojani (Asch. \& Sint. ex Boiss.) Coode \& Cullen, Pinus sylvestris L, Carpinus betulus L., 994 m, 15.10.2017.

10-Karabük, Eflani, Akçakese Village, Dere Mah. vicinity, $41^{\circ} 32^{\prime} 079^{\prime \prime} \mathrm{N}$ 032 52'38.1"E, Abies nordmanniana subsp. equi-trojani (Asch. \& Sint. ex Boiss.) Coode \& Cullen, $710 \mathrm{~m}$, 15.10.2017.

11-Karabük, Eflani, Çalı Kahvesi, 4120'04.8"N $032^{\circ} 52^{\prime} 11.5^{\prime \prime E}$, open areas and Pinus sylvestris L, Cornus mas L., Quercus sp. L., $860 \mathrm{~m}$, 31.03.2018.

12-Karabük, Eflani, Çukurören village vicinity, $41^{\circ} 21^{\prime} 01.8^{\prime \prime N} 032^{\circ} 52^{\prime} 01.8^{\prime \prime} \mathrm{E}$, Carpinus betulus L., Quercus sp. L., 992 m, 31.03.2018.

13-Karabük, Eflani, Değirmendere vicinity, $41^{\circ} 20^{\prime} 51.9^{\prime \prime} \mathrm{N} 032^{\circ} 53^{\prime} 12.5^{\prime \prime E}$, Carpinus betulus L., Alnus glutinosa (L.) Gaertn., Salix sp. L., Quercus sp. L., Juniperus sp. L., Cornus mas L., $850 \mathrm{~m}, 31.03 .2018$.

14-Karabük, Eflani, Bozarmut village, $41^{\circ} 19^{\prime} 16.8^{\prime \prime} \mathrm{N} \quad 032^{\circ} 54^{\prime} 46.2^{\prime \prime} \mathrm{E}$, farmland and Pinus nigra subsp. pallasiana (Lamb.) Holmboe, Quercus sp. L., 1036 m, 31.03.2018.

15-Karabük, Eflani, Çal village vicinity, $41^{\circ} 21^{\prime} 35.3^{\prime \prime} \mathrm{N} \quad 032^{\circ} 56^{\prime} 32.0^{\prime \prime} \mathrm{E}$, open areas, Quercus sp. L., 1036 m, 01.04.2018.

16-Karabük, Eflani, between Tabaklar stream and Çalışlar village, $41^{\circ} 23^{\prime} 33.9^{\prime \prime} \mathrm{N}$ 032 $56^{\circ} 08.3^{\prime \prime} \mathrm{E}$, rocky steam banks, Quercus sp., 890 m, 01.04.2018.

17-Karabük, Eflani, Çal village, Kızılgelik vicinity, $41^{\circ} 20^{\prime} 56.5^{\prime \prime} \mathrm{N} \quad 032^{\circ} 58^{\prime} 23.8^{\prime \prime} \mathrm{E}$, open areas, Quercus sp. L., 1054 m, 01.04.2018.

18-Karabük, Eflani, between Gelicek and Karacapınar, $\quad 41^{\circ} 23^{\prime} 13.7^{\prime \prime} \mathrm{N} \quad 033^{\circ} 00^{\prime} 23.9^{\prime \prime} \mathrm{E}$, Populus tremula L., Pinus sylvestris L, Pinus nigra subsp. pallasiana (Lamb.) Holmboe, Quercus sp. L., Carpinus betulus L., open areas, $970 \mathrm{~m}, 01.04 .2018$.

19-Karabük, Eflani, Akçakese vicinity, $41^{\circ} 32^{\prime} 07,7^{\prime \prime} \mathrm{N} \quad 32^{\circ} 52^{\prime} 54,5^{\prime \prime} \mathrm{E}, \quad$ Abies nordmanniana subsp. equi-trojani (Asch. \& Sint. ex Boiss.) Coode \& Cullen, Carpinus betulus L., Pinus nigra subsp. pallasiana (Lamb.) Holmboe, Salix alba L., 740 m, 06.04.2019.

20-Karabük, Eflani, Ovaçalış village, 41 ${ }^{\circ} 31^{\prime 2} 24,9^{\prime \prime} \mathrm{N}$ $32^{\circ} 55^{\prime} 01,6 " \mathrm{E}$, old farmland, Cornus mas L., Crataegus sp. L., Quercus sp. L., 800 m, 06.04.2019.

21-Karabük, Eflani, Bağlıca village, Çelebioğlu vicinity, $41^{\circ} 33^{\prime} 06.0^{\prime \prime} \mathrm{N} 32^{\circ} 58^{\prime} 50,5^{\prime \prime} \mathrm{E}$, open areas 
and Carpinus betulus L., Fagus orientalis Lipsky, Pinus nigra subsp. pallasiana (Lamb.) Holmboe, Quercus sp. L., Populus tremula L., Pyracantha coccinea M.Roem., $1005 \mathrm{~m}$, 06.04.2019.

22-Karabük, Eflani, Soğucak Hill, wetlands, $41^{\circ} 30^{\prime} 11.7^{\prime \prime} \mathrm{N} 32^{\circ} 57^{\prime} 10.2^{\prime \prime} \mathrm{E}$, Populus tremula L., Pinus nigra subsp. pallasiana (Lamb.) Holmboe, Pinus sylvestris L., Cornus mas L., Juniperus sp. L., 1140 m, 06.04.2019.

23-Karabük, Eflani, Çukurgelik vicinity, $41^{\circ} 30^{\prime} 43.5^{\prime \prime} \mathrm{N} 32^{\circ} 59^{\prime} 17.5^{\prime \prime E}$, Fagus orientalis Lipsky, Quercus sp. L., Carpinus betulus L., Cornus mas L., Crataegus sp. L., $1120 \mathrm{~m}$, 07.04.2019.

24-Karabük, Eflani, Kadıköy Pond, 4127'42.3"N $33^{\circ} 00^{\prime} 22.2^{\prime \prime E}$, wetlans, $946 \mathrm{~m}, 07.04 .2019$.

25-Karabük, Eflani, Esencik Koruboğazı Stream vicinity, $41^{\circ} 29^{\prime} 03.9^{\prime \prime} \mathrm{N} \quad 33^{\circ} 01^{\prime} 50.4^{\prime \prime E}$, Pinus nigra subsp. pallasiana (Lamb.) Holmboe, Pinus sylvestris L., Juniperus sp. L., Quercus sp. L. 1002 m, 07.04.2019.

26-Karabük, Eflani, Bostancilar Pond, $41^{\circ} 27^{\prime} 28.0^{\prime \prime} \mathrm{N} 32^{\circ} 57^{\prime} 06.4^{\prime \prime} \mathrm{E}$, wetland, Salix sp. L., $945 \mathrm{~m}, 07.04 .2019$.

27-Karabük, Eflani, Bedil village vicinity,

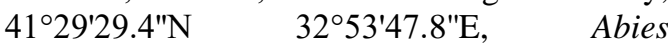
nordmanniana subsp. equi-trojani (Asch. \& Sint. ex Boiss.) Coode \& Cullen, Cornus mas L., Fagus orientalis Lipsky, Corylus avellana L., $1090 \mathrm{~m}, 07.04 .2019$.

28-Karabük, Eflani, Hacıağaç village vicinity, $41^{\circ} 28^{\prime} 22.2^{\prime \prime} \mathrm{N} \quad 32^{\circ} 52^{\prime} 00.3^{\prime \prime} \mathrm{E}, \quad$ Abies nordmanniana subsp. equi-trojani (Asch. \& Sint. ex Boiss.) Coode \& Cullen, Fagus orientalis Lipsky, 945 m, 07.04.2019.

29-Karabük, Eflani, Hacıağaç village forest, $41^{\circ} 27^{\prime} 55.5^{\prime \prime} \mathrm{N} \quad 32^{\circ} 51^{\prime} 33.7^{\prime \prime} \mathrm{E}, \quad$ Abies nordmanniana subsp. equi-trojani (Asch. \& Sint. ex Boiss.) Coode \& Cullen, Fagus orientalis Lipsky, 993 m, 07.04.2019.

30-Karabük, Eflani, Kavak village vicinity, 4126'01.9"N 3254'56.4"E, Pinus sylvestris L., Quercus sp. L., Cornus mas L., Juniperus sp. L., $977 \mathrm{~m}, 07.04 .2019$.

Diagnostic characters of specimens were examined by using stereo and light microscope, and specimens were identified by related revision, monographs, and floristic books (Smith, 1996, 2004; Paton, 1999; Guerra et al., 2006; Cortini Pedrotti, 2001, 2006; Greven, 2003; Lara et al., 2009; Casas et al., 2006, 2009; Kürschner and Frey, 2020).

Bryofloristic studies conducted in the area (Özalp, 1995; Uyar, 2003; Keçeli and Çetin, 2006; Uyar and Çetin, 2006; Uyar et al., 2007; Ursavaş and Abay,
2009; Ören et al., 2015; Sar1 and Ören, 2016) and the distribution of the bryophyte species in Turkey were reviewed for determining the new bryophyte taxa for Henderson's A2 Grid and the Karabük (Kürschner and Erdağ, 2021). Families and upper taxonomic categories in the floristic list are arranged according to Hodgetts et al. (2020)'s systematically hierarchy, and species are listed alphabetically under families in the bryofloristic list given in the appendix.

All specimens deposited at Zonguldak Bülent Ecevit University, Bryophyte Herbarium (ZNG).

\section{Result and Discussion}

156 specific and infraspecific bryophyte taxa (15 liverworts and 141 mosses) have been identified with the examination of approximately 500 bryophyte specimens collected from 30 different localities in the study area. Lewinskya acuminata (H.Philib.) F.Lara, Garilleti \& Goffinet is new to Henderson's A2 sqaure and nineteen species newly reported from Karabük province. These species are Riccardia chamedryfolia (With.) Grolle, Riccia beyrichiana Hampe, Polytrichum juniperinum Hedw., Polytrichum piliferum Hedw., Fissidens adianthoides Hedw., Pleuridium subulatum (Hedw.) Rabenh., Gymnostomum calcareum Nees \& Hornsch., Syntrichia latifolia (Schwägr.) Venturi ex Broth., Syntrichia papillosissima (Copp.) Loeske, Tortula schimperi M.J.Cano, O.Werner \& J.Guerra, Grimmia laevigata (Brid.) Brid., Racomitrium elongatum Ehrh. ex Frisvoll, Racomitrium heterostichum (Hedw.) Brid., Schistidium elegantulum H.H.Blom, Ptychostomum kunzei (Hornsch.) J.R.Spence, Lewinskya acuminata (H.Philib.) F.Lara, Garilleti \& Goffinet, Plagiothecium curvifolium Schlieph. ex Limpr., Drepanocladus aduncus (Hedw.) Warn, Leptodictyum riparium (Hedw.) Warnst..

There is no previous comprehensive study on the bryophyte diversity of Eflani District. However, some bryophyte records were given by Ören et al. (2012) and Arslan et al. (2018) from only 4 localities within the borders of Eflani District. These species are Lophocolea bidentata (L.) Dumort., Metzgeria furcata (L.) Dumort., Ptilidium pulcherrimum (Weber) Vain., Riccia cavernosa Hoffm. from liverworts and Buxbaumia viridis (Moug. ex Lam. \& DC.) Brid. ex Moug. \& Nestl., Calliergonella cuspidata (Hedw.) Loeske., Dicranum tauricum Sapjegin, Ephemerum minutissimum Lindb., Physcomitrium patens Hedw., Plagiochila porelloides (Torrey ex Nees) Lindenb., Plagiomnium undulatum (Hedw.) T.J.Kop., Plagiothecium denticulatum (Hedw.) Schimp., Rhizomnium punctatum (Hedw.) 
T.J.Kop., Sanionia uncinata (Hedw.) Loeske from mosses. Considering the previous studies together with this study, the bryophyte diversity reached up to 160 in Eflani District, and 336 in Karabük Province (Keçeli and Çetin, 2006; Uyar and Çetin, 2006; Ören et al., 2012; Ören et al., 2015; Sar1 ve Ören, 2016; Arslan et al., 2018; Verimbaş, 2019).

Note: This study was presented as an oral presentation in EurasianBioChem 2020 Conference and published as a summary text in the conference proceedings book.

\section{References}

Abay G. Erata H. Batan N. Özdemir T. 2021. Two new records for the bryophyte flora of Turkey and Southwest Asia, Plant Biosystems - An International Journal Dealing with all Aspects of Plant Biology, DOI: 10.1080/11263504.2021.1947407

Akman Y. 1999. İklim ve Biyoiklim (Biyoiklim Metodları ve Türkiye İklimleri). Kariyer Matbaacilik. Ankara.

Arslan A. Unan A.D. Ören M. 2018. A new locality for two remarkable bryophytes in Turkey. Anatolian Bryology. 4:1, 1-7.

Casas C. Brugués M. Cros M.R. Sérgio C. 2006. Handbook of Mosses of the Iberian Peninsula and The Balearic Islands, Institut D'estudis Catalans. Barcelona, 349 p.

Casas C. Brugués, M. Cros, M.R. Sérgio C. Infante M. 2009. Handbook of Liverworts and Hornworts of the Iberian Peninsula and The Balearic Islands, ISBN: 978-84-92583-553, 177 p, Institut D'estudis Catalans, Spain.

Cortini Pedrotti C. 2001. Flora dei muschi d"eItalia, Sphagnopsida, Andreaopsida, Bryopsida (I parte). ISBN: 88-7287-250-2, Antonio Delfino Editore Medicina-Scienze.

Cortini Pedrotti C. 2006. Flora dei muschi de'Italia, Bryopsida (II parte), ISBN: 88-7287-370-3, Antonio Delfino Editore Medicina-Scienze, Roma.

Erata H. Batan H. 2020. New and remarkable bryophyte records from Turkey and SouthWest Asia, Plant Biosystems - An International Journal Dealing with all Aspects of Plant Biology. 154:3, 376-383

Erata H. Özen Ö. Batan N. Alatas M. 2021. Pohlia Hedw. and Oleolophozia L.Söderstr., De Roo \& Hedd. species new to Turkey and South-West Asia. Cryptogamie, Bryologie. 42:1 1-10.

Glime J.M. 2017. Bryophyte Ecology. Ebook sponsored by Michigan Technological University and the International Association of Bryologists. Last updated 18 July 2020 and available
$<$ https://digitalcommons.mtu.edu/bryophyte -ecology/>.

Gözcü C.M. Uyar G. Ören M. Ezer T. Alataş M. 2019. The bryophyte flora of the Samanlı Mountains (Sakarya, Kocaeli, Yalova, Bursa) in North-West Turkey. Arctoa. 28: 58-7.

Greven H.C. 2003. Grimmias of the World. Backhuys Publishers, Leiden, 247 pp.

Guerra J. Cano M.J. Cros R.M. 2006. Flora Briofitica Ibérica Volume 3, Uniersidad de Murcia, ISBN: 84-609-9097-4. Sociedad Espanola de Briyologia Murcia. Murcia.

Hodgetts N.G. Söderström L. Blockeel T.L. Caspari S. Ignatov M.S. Konstantinova N.A. Lockhart N. Papp B. Schröck C. Sim-Sim M. Bell D. Bell N.E. Blom H.H. BruggemanNannenga M.A. Brugués M. Enroth J. Flatberg K.I. Garilleti R. Hedenäs L. Holyoak D.T. Hugonnot V. Kariyawasam I. Köckinger H. Kučera J. Lara F. Porley R.D. 2020. An annotated checklist of bryophytes of Europe, Macaronesia and Cyprus. Journal of Bryology. 42:1, 1-116.

Keçeli T. Çetin B. 2006. A Contribution to the Liverwort Flora of Western Black Sea Region, Northern Turkey, and a new record (Cephaloziella dentata, Cephaloziellaceae) to Southwest Asia. Cryptogamie Bryologie. 27:4, 459-470.

Kürschner H. Erdağ A. 2021. Bryophyte locality data from the Near and Middle East 17752019 (Afghanistan, Bahrain, Iran, Iraq, Israel, Jordan, Kuwait, Lebanon, Oman, Qatar, Saudi Arabia, Sinai Peninsula, Syria, Turkey, United Arab Emirates and Yemen (incl. Socotra)) 1:6, Hiperlink Yayınları, İstanbul.

Kürschner H. Frey W. 2020. Liverworts, Mosses and Hornworts of Southwest Asia, Nowa Hedwigia, Beiheft 149, J. Cramer in Borntraeger Science Publishers, Stuttgart, $267 \mathrm{pp}$.

Kürschner H. Frey W. 2020. Liverworts, Mosses and Hornworts of Southwest Asia, Nowa Hedwigia, Beiheft 149, J. Cramer in Borntraeger Science Publishers, Stuttgart, $267 \mathrm{pp}$.

Lara F. Garilleti R. Medina R. Mazımpaka V. 2009. A new key to the Orthotrichum Hedw. in Europe and the Mediterranean Region. Cryptogamie Bryologie. 30:1, 129-142.

Ören M. Sarı B. Ursavaş S. 2015. Syntrichia minor (Pottiaceae) and Cephaloziella integerrima (Cephaloziellaceae) new to bryophyte flora of Turkey. Archives of Biological Sciences. 67:2, 367-372. 
Ören M. Uyar G. Keçeli T. 2012. The bryophyte flora of the western part of the Küre Mountains (Bartın, Kastamonu). Turkey. Turkish Journal of Botany. 36: 538-557.

Özalp G. 1995. Çitdere Bölgesi (Yenice, Zonguldak)'nin Kriptogam Florasına Katk1. IÜ Orman Fakültesi Dergisi, Ayrı Bask1, Seri A. 45:1, 35-43.

Özenoğlu H. Kırmacı M. Kiremit F. 2019. Contributions to the genus Riccia L. (Ricciaceae) in Turkey. Turkish Journal of Botany. 43:2, 253-261.

Paton J. 1999. The Liverworts Flora of the British Isles, 626 pp, Harley Books, England.

Sarı B. Ören M. 2016. Safranbolu İlçesi (Karabük) Briyofit Floras1. Kastamonu University Journal of Forestry Faculty. 16:1, 157-168.

Smith A.J.E. 1996. The Liverworts of Britain and Ireland, ISBN: 0-521-42473-9, 384 pp Cambridge University Press, Cambridge.

Smith A.J.E. 2004. The Moss Flora of Britain and Ireland. Second Edition, ISBN: 0-21816408, 1012 pp, Cambridge University Press, Cambridge.

Unan A.D. Ören M. 2021. New and noteworthy records of deadwood dwelling bryophyte species for Turkey and Southwest Asia. Cryptogamie, Bryologie. 42:4, 33-44.

Unan A.D. Potemkin A. Ursavaş S. Çalışkan S. Ören M. 2021. New records of two Scapania species (Scapaniaceae, Marchantiophyta) from north of Turkey, Plant Biosystems - An International Journal Dealing with all Aspects of Plant Biology. 155:4, 679-684.

URL 1. TC Karabük Valiliği, Eflani ilçesi, 2021. http://www.karabuk.gov.tr/eflani-ilcesi [Erişim: 08 Eylül 2021].

URL 2. TC Eflani Kaymakamlığı, 2021. http://www.eflani.gov.tr/ilcemizdengorunum. [Erişim: 08 Eylül 2021].

URL 3. TC Maden Teknik ve Arama Genel Müdürlüğü, <https://www.mta.gov.tr/v3.0/hizmetler/50 Obas>, [Erişim: 15 Aralık 2020].

Ursavaş S. Abay G. 2009. Türkiye'nin A2 Karesinin Karayosunları (Musci) Kontrol Listesi. Bartın Orman Fakültesi Dergisi. 11:16, 33-43.

Ursavaş S. Işın Z. 2019. New records of Bryum gemmiferum and Atrichum crispum for Turkey. Plant Biosyst. 153:5, 686-690.

Ursavaş S. Keçeli T. 2019. Weissia multicapsularis, a rare moss species new to Turkey and Asia, Plant Biosystems - An International Journal Dealing with all Aspects of Plant Biology. 153:5, 669-672

Ursavaş S. Keçeli T. Uyar G. Ören M. 2021. Dicranella staphylina (Dicranaceae), a new moss record from Turkey and South West Asia, Plant Biosystems - An International Journal Dealing with all Aspects of Plant Biology, 155:3, 483-486.

Uyar G. 2003. Two New Varieties of Ctenidium molluscum (Hedw.) Mitt. (Hypnaceae, Musci) for The Moss Flora of Turkey. Turkish Journal of Botany. 27: 227-229.

Uyar G. Alataş M. Ören M. Keçeli T. 2007. The Bryophyte Flora of Yenice Forests (Karabük, Turkey). International Journal of Botany. 3:2, 129-146.

Uyar G. Çetin B. 2006. Contribution to the Moss Flora of Turkey: Western Black Sea Region (Bolu, Katamonu, Karabük, Bartın and Zonguldak). International Journal of Botany. 2:3, 229-241.

Verimbaş B. 2019. Ovacık (Karabük) İlçesi Briyofit Floras1, (Yüksek lisans tezi), Zonguldak Bülent Ecevit Üniversitesi, Fen Bilimleri Ensitüsü, Biyoloji Anabilim Dalı, Zonguldak, 91s. 
Appendix

Bryofloristic List

MARCHANTIOPHYTA Stotler \& Crand. Stotl.

JUNGERMANNIOPSIDA Stotler \& Crand. Stotl. JUNGERMANNIALES H.Klinggr

Cephaloziaceae Mig.

1. Cephalozia ambigua (L.) Dumort. - 1, on soil, AArslan 160.

2. Nowellia curvifolia (Dicks.) Mitt - 19, on deadwood, AArslan 38.

Cephaloziellaceae Douin

3. Cephaloziella divaricata (Sm.) Schiffn - 1, 19, 28, on soil and deadwood, AArslan 45.

Blepharostomataceae W.Frey \& M.Stech

4. Blepharostoma trichophyllum (L.) Dumort. - 19 , on deadwood, AArslan 183.

Lophocoleaceae Vanden Berghen

5. Lophocolea heterophylla (Schrad.) Dumort. - 19, 28, on deadwood, AArslan 46.

Plagiochilaceae Müll. Frib.

6. Plagiochila porelloides (Torr. ex Nees) Lindenb. - 6, 19, on soil and rocks, AArslan 184.

PORELLALES Schljakov

Frullaniaceae Lorch

7. Frullania dilatata (L.) Dumort. - 1, 2, 11, 18, on tree bark and rocks, AArslan 246.

Porellaceae Cavers

8. Porella platyphylla (L.) Pfeiff. - 1, 9, on bark, AArslan 158

Radulaceae Müll.Frib.

9. Radula complanata (L.) Dumort. - 1, 2, 11, 15, 17, on tree bark and rocks, AArslan 217.

METZGERIALES Chalaud

Aneuraceae H.Klinggr

10. Aneura pinguis (L.) Dumort. - 22, on wet dead wood, AArslan 58.

11. *Riccardia chamedryfolia (With.) Grolle - 19, on wet soil at stream bank, AArslan 32.

PELLIALES He-Nygrén

Pelliaceae H.Klinggr.

12. Apopellia endiviifolia (Dicks.) Nebel \& D.Quandt (Syn: Pellia endiviifolia (Dicks.) Dumort) - 8, 10, on wet soil, AArslan 144.

MARCHANTIOPSIDA Gonquist, Takht \& W.Zimm.

MARCHANTIALES Limpr.

Ricciaceae Rchb.

13. *Riccia beyrichiana Hampe - 11, on soil at open area, AArslan 120, 204.

14. Riccia cavernosa Hoffm. - 5, on wet soil near ponds, AArslan 205.

15. Riccia gougetiana Durieu \& Mont. - 11, on soil at open area, AArslan 106.

BRYOPHYTA Schimp.

POLYTRICHOPSIDA Doweld

POLYTRICHALES M.Fleisch.

Polytrichaceae Schwägr
16. Artichum undulatum (Hedw.) P.Beauv. - 1, 8, on rocks and soil, AArslan 249.

17. Polytrichum formosum Hedw. (Syn: Polytrichastrum formosum (Hedw.) G.L.Sm.) - 8, on soil, AArslan 297.

18. *Polytrichum juniperinum Hedw. - 1, on soil, AArslan 308.

19. *Polytrichum piliferum Hedw. - 1, 11, on soil, AArslan 148.

20. Pogonatum urnigerum (Hedw.) P.Beauv. - 1, on soil, AArslan 296.

BRYOPSIDA Pax

BUXBAUMIALES M.Fleisch.

Buxbaumiaceae Schimp.

21. Buxbaumia viridis (Moug. ex Lam. \& DC.) Brid. ex Moug. \& Nest. - 9, 28, on deadwood, AArslan 33.

ENCALYPTALES Dixon

Encalyptaceae Schimp.

22. Encalypta streptocarpa Hedw. - 1, 6, 12, 16, 25 , on rocks, AArslan 149.

23. Encalypta rhaptocarpa Schwägr. - 16, on rocks, AArslan 309.

24. Encalypta vulgaris Hedw. - 16, on soil covered rocks, AArslan 228.

FUNARIALES M.Fleisch.

Funariaceae Schwägr.

25. Funaria hygrometrica Hedw. - 16, on soil, AArslan 128.

26. Physcomitrium patens (Hedw.) Mitt. (Syn: Aphanorrhegma patens (Hedw.) Lindb., Physcomitrella patens (Hedw.) Bruch \& Schimp.) - 5, on wet soil near ponds, AArslan 207A.

DICRANALES H.Philib. ex M.Fleisch

Flexitrichaceae Ignatov \& Fedosov

27. Flexitrichum flexicaule (Schwägr.) Ignatov \& Fedosov (Syn: Ditrichum flexicaule (Schwägr.) Hampe) - 1, 11, on rocks and soil, AArslan 187.

Dicranellaceae M.Stech

28- Dicranella howei Renauld \& Cardot - 7, on soil,

29. Dicranella varia (Hedw.) Schimp. - 8, 10, 22, on soil, AArslan 151.

Fissidentaceae Schimp.

30. *Fissidens adianthoides Hedw. - 1, on soil, AArslan 220.

31. Fissidens dubius P.Beauv. - 6, 9, 16, on rocks and soil, AArslan 220.

32. Fissidens taxifolius Hedw. - 1, 9, 18, 28, on soil and rocks, AArslan 166.

Dicranaceae Schimp.

33. Dicranum scoparium Hedw. - 1, 9, 12, 15, 17, 23, 25, 29, On soil, rocks, bark, roots, deadwood, AArslan 142.

34. Dicranum tauricum Sapjegin - 1, 9, on deadwood, AArslan 170.

Ditrichaceae Limpr. 
35. Pleuridium acuminatum Lindb. - 1, on soil, AArslan 310.

36. *Pleuridium subulatum (Hedw.) Rabenh. - 1, on soil, AArslan 311.

Pottiaceae Schimp.

37. Barbula unguiculata Hedw. - 1, 3, 8, 10, 12, 13, on soil and rock crevices, AArslan 251.

38. Bryoerythrophyllum recurvirostrum (Hedw.) P.C.Chen - 13, on roots near streams,

39. Cinclidotus fontinaloides (Hedw.) P.Beauv. 13, on submerged rocks, AArslan 139.

40. Cinclidotus riparius (Host ex Brid.) Arn. İstasyon 13, on submerged rocks, AArslan 109.

41. Didymodon acutus (Brid.) K.Saito - 3, 16, on soil, AArslan 155.

42. Didymodon luridus Hornsch. - 3, 8, 10, 14, on soil and rocks, AArslan 290.

43. Didymodon nicholsonii Culm. - 13, on wet deadwood near stream, AArslan 306.

44. Didymodon tophaceus (Brid.) Lisa - 11, on concrete, AArslan 320.

45. Didymodon vinealis (Brid.) R.H.Zander - 12, on soil, AArslan 355.

46. *Gymnostomum calcareum Nees \& Hornsch. 16, on wet calcareous rocks, AArslan 125.

47. Streblotrichum convolutum (Hedw.) P.Beauv. (Syn: Barbula convoluta Hedw.) - 11, on soil, AArslan 295.

48. *Syntrichia latifolia (Schwägr.) Venturi ex Broth. - 8, 13, on roots and barks near streams, AArslan 250.

49. *Syntrichia papillosissima (Copp.) Loeske - 25, on rocks at open lands, AArslan 62.

50. Syntrichia ruralis (Hedw.) F.Weber \& D.Mohr - $1,8,9,12,14$, on soil, rocks and barks, AArslan 2.

51. Syntrichia virescens (De Not.) Ochyra - 13, on barks, AArslan 133

52. Tortella inclinata (R.Hedw.) Limpr. - 11, on soil, AArslan 127.

53. Tortella squarrosa (Brid.) Limpr. (Syn: Pleurochaete squarrosa (Brid.) Lindb.) - 5, 11, on soil and rocks, AArslan 91.

54. Tortella tortuosa (Hedw.) Limpr. - 2, 5, 6, 12, 25, on soil and rocks,AArslan 293.

55. Tortula inermis (Brid.) Mont. - 1, on soil, AArslan 356.

56. Tortula muralis Hedw. - 10, 11, on rocks and concrete, AArslan 288.

57. *Tortula schimperi M.J.Cano, O.Werner \& J.Guerra - 1, on soil, AArslan 141.

58. Tortula subulata Hedw. - 12, 13, on soil, AArslan 19.

59. Trichostomum brachydontium Bruch - 1, on soil, AArslan 382.

60. Trichostomum crispulum Bruch - 11, 14, 22, on oil, AArslan 179.
61. Weissia brachycarpa (Nees \& Hornsch.) Jur. 14, on soil, AArslan 389.

62. Weissia condensa (Voit) Lindb. - 1, 14, 20, on soil, AArslan 273.

63. Weissia controversa Hedw. - 1, 16, on soil, AArslan 196.

GRIMMIALES M.Fleisch.

Seligeriaceae Schimp.

64. Seligeria acutifolia Lindb. - 6, 16, on rocks, AArslan 277.

65. Seligeria recurvata P.Beauv. - 28 , on rocks, AArslan 392.

Grimmiaceae Arn.

66. *Grimmia laevigata (Brid.) Brid. - 16, on rocks, AArslan 402.

67. Grimmia pulvinata (Hedw.) Sm. - 1, 8, 11, 18, 25, on rocks and concrete, AArslan 61.

68. Grimmia tergestina Tomm. ex Bruch \& Schimp. - 16, on rocks, AArslan 413.

69. *Racomitrium elongatum Ehrh. ex Frisvoll - 1, 8, on soil, AArslan 269.

70. *Racomitrium heterostichum (Hedw.) Brid. 1, on rocks, AArslan 248.

71. Schistidium apocarpum (Hedw.) Bruch \& Schimp. - 2, 8, 13, 17, on rocks and roots, AArslan 278.

72. Schistidium crassipilum H.H.Blom - 1, 9, 12, 13, 18, on rocks, AArslan 240.

73. *Schistidium elegantulum H.H.Blom - 10, on rocks, AArslan 214.

74. Schistidium helveticum (Schkuhr) Deguchi (Syn: Schistidium singarense (Schiffn.) Laz.) - 11, on concrete, AArslan 414.

HEDWIGIALES Ochyra

Hedwigiaceae Schimp.

75. Hedwigia ciliata (Hedw.) P.Beauv. - 1, on rocks, AArslan 266.

BRYALES Limpr.

Bryaceae Schwägr

76. Bryum argenteum Hedw. - 20, on rocks, AArslan 75.

77. Imbribryum alpinum (Huds. ex With.) N.Pedersen (Syn: Bryum alpinum Huds. ex With.) - 8, 16, on soil, AArslan 254.

78. Ptychostomum capillare (Hedw.) Holyoak \& N.Pedersen (Syn: Bryum capillare Hedw.) - 6, $12,23,25$, on rocks and soil, AArslan 55.

79. Ptychostomum imbricatulum Holyoak \& N.Pedersen (Syn: Bryum caespiticium Hedw.) - 3, 12, 14, on soil, AArslan 176.

80. *Ptychostomum kunzei (Hornsch.) J.R.Spence (Syn: Bryum kunzei Hornsch.)

- 14, on soil, AArslan 27.

81. Ptychostomum moravicum (Podp.) Ros \& Mazimpaka (Syn: Bryum moravicum Podp.) $6,7,8,10,12,13,17,21$, on rocks, soil and barks, AArslan 188. 
82. Ptychostomum pallens (Sw. ex anon.) J.R.Spence (Syn: Bryum pallens Sw. ex anon.) - 22, on wet wood and soil, AArslan 198.

83. Ptychostomum pseudotriquetrum (Hedw.) J.R.Spence \& H.P.Ramsay ex Holyoak \& N.Pedersen (Syn: Bryum neodamense Itzigs., Bryum pseudotriquetrum (Hedw.) P.Gaertn. B.Mey. \& Scherb.) - 11, 22, on wet soil, AArslan 11.

84. Ptychostomum torquescens (Bruch \& Schimp.) Ros \& Mazimpaka (Syn: Bryum torquescens Bruch \& Schimp.) - 16, on soil, AArslan 305.

85. Rhodobryum roseum (Hedw.) Limpr. - 6, on soil, AArslan 93.

Mniaceae Schwägr

86. Mnium hornum Hedw. - 6, on rocks, AArslan 236.

87. Mnium stellare Hedw. - 7, on rocks, AArslan 164.

88. Plagiomnium affine (Blandow ex Funck) T.J.Kop. - 8, on soil, AArslan 235.

89. Plagiomnium elatum (Bruch \& Schimp.) T.J.Kop. - 17, on roots, AArslan 227.

90. Plagiomnium undulatum (Hedw.) T.J.Kop. - 8, 9, on soil, AArslan 88.

91. Pohlia wahlenbergii (F.Weber \& D. Mohr) A.L.Andrews - 10, on wet soil, AArslan 123.

92. Rhizomnium punctatum (Hedw.) T.J.Kop. - 9, 19, on wet soil and deadwood, AArslan 84.

ORTHOTRICHALES Dixon

Orthotrichaceae Arn

93. **Lewinskya acuminata (H.Philib.) F.Lara, Garilleti \& Goffinet - 18, on barks, AArslan 257.

94. Lewinskya affinis (Schrad. ex Brid.) F.Lara, Garilleti \& Goffinet (Syn: Orthotrichum affine Schrad.ex Brid.) - 1, 5, 11, on barks, AArslan 121.

95. Lewinskya sordida (Sull. \& Lesq.) F.Lara, Garilleti \& Goffinet (Syn:Orthotrichum sordidum Sull. \& Lesq.) - 2, on roots, AArslan 268.

96. Lewinskya speciosa (Nees) F.Lara, Garilleti \& Goffinet (Syn: Orthotrichum speciosum Nees) - 1, 5, 8, 9, 11, 15, 18, on barks, AArslan 303.

97. Lewinskya striata (Hedw.) F.Lara, Garilleti \& Goffinet (Syn: Orthotrichum striatum Hedw.) - 1, 5, 9, 18, 20, on barks, AArslan 222.

98. Orthotrichum anomalum Hedw. - 12, on rocks, AArslan 224.

99. Orthotrichum cupulatum Brid. - 13, on rocks and roots near stream, AArslan 192.

100. Orthotrichum pumilum Sw. ex anon. - 11, 13, on barks, AArslan 211.

101. Pulvigera lyellii (Hook. \& Taylor) Plášek, Sawicki \& Ochyra (Syn: Orthotrichum lyellii Hook. \& Taylor) - 9, 11, on barks, AArslan 216.
HYPNALES W.R.Buck \& Vitt

Fontinalaceae Schimp.

102. Fontinalis antipyretica Hedw. - 13, on submerged rocks, AArslan 136.

Plagiotheciaceae M.Fleisch.

103. Herzogiella seligeri (Brid.) Z.Iwats. - 6, 19, 28, on deadwood, AArslan 282.

104. *Plagiothecium curvifolium Schlieph. ex Limpr. - 28, on deadwood, AArslan 199.

105. Plagiothecium denticulatum (Hedw.) Schimp. - 8, 9, on roots, barks and soil, AArslan 108.

Pterigynandraceae Schimp

106. Pterigynandrum filiforme Hedw. - 1, 8, 11, on barks, AArslan 194.

Amblystegiaceae G.Roth.

107. Campyliadelphus chrysophyllus (Brid.) R.S.Chopra - 2, 11, 22, on rocks and soil, AArslan 264.

108. Campylophyllopsis calcarea (Crundw. \& Nyholm) Ochyra (Syn: Campylidium calcareum (Crundw. \& Nyholm) Ochyra, Campylophyllum calcareum (Crundw. \& Nyholm) Hedenäs) - 5, 13, on wet calcareous rocks, AArslan 168.

109. Campylium protensum (Brid.) Kindb. - 18, on rocks, AArslan 238.

110. Cratoneuron filicinum (Hedw.) Spruce - 3, 8, 10, 11, 22, on wet soil and rocks, AArslan 24.

111. *Drepanocladus aduncus (Hedw.) Warn - 21, 22, 24, on wet soil and submerged, AArslan 77.

112. *Leptodictyum riparium (Hedw.) Warnst. - 8, on damp barks, AArslan 292.

Scorpidiaceae Ignatov \& Ignatova

113. Sanionia uncinata (Hedw.) Loeske - 8, on damp barks, AArslan 147 A, B ve C.

Leskeaceae Schimp.

114. Leskea polycarpa Hedw. - 13, on wet rocks near stream, AArslan 428.

Thuidiaceae Schimp

115. Abietinella abietina (Hedw.) M.Fleisch. - 2, 4, 23, on soil, AArslan 263.

116. Thuidium assimile (Mitt.) A.Jaeger - 19, on rocks, AArslan 201.

117. Thuidium delicatulum (Hedw.) Schimp. - 8, on soil, AArslan 245.

118. Thuidium tamariscinum (Hedw.) Schimp. - 8, on soil and roots, Arslan 243.

Brachytheciaceae G.Roth.

119. Brachytheciastrum salicinum (Schimp.) J.D.Orgaz, M.J.Cano \& J.Guerra (Syn: $B$. velutinum Hedw.) Ignatov \& Huttunen var. salicinum (Schimp.) Ochyra \& Zarnowiec) - 1, 5,28 , on tree trunk, on deadwood and rocks, AArslan 294. 
120. Brachytheciastrum velutinum (Hedw.) Ignatov \& Huttunen - 1, on soil and rocks, AArslan 110.

121. Brachythecium albicans (Hedw.) Schimp. - 3, on soil, AArslan 98.

122. Brachythecium glareosum (Bruch ex Spruce) Schimp. - 2, 4, on soil, AArslan 281.

123. Brachythecium mildeanum (Schimp.) Schimp. - 21, on damp and wet soil near forest, AArslan 42.

124. Brachythecium rivulare Schimp. - 13, 17, 18, on wet rocks, soil and roots near stream, AArslan 241.

125. Brachythecium rutabulum (Hedw.) Schimp. 8, 19, on bark and rocks, AArslan 175.

126. Eurhynchium striatum (Hedw.) Schimp. - 9, 19, 28, on soil and roots, AArslan 82, 92.

127. Homalothecium lutescens (Hedw.) H.Rob. 1, kaya, on bark and soil, AArslan 169.

128. Homalothecium sericeum (Hedw.) Schimp. 2, 6, 19, 23, on rocks, soil and bark, AArslan 280.

129. Microeurhynchium pumilum (Wilson) Ignatov \& Vanderp. - 8, 19, on damp soil and roots, AArslan 31.

130. Oxyrrhynchium hians (Hedw.) Loeske - 7, on soil and rocks, AArslan 103.

131. Oxyrrhynchium schleicheri (R.Hedw.) Röll 13, on soil at forest road slope, AArslan 427.

132. Pseudoscleropodium purum (Hedw.) M.Fleisch. - 1, 11, 15, 25, 29, 30, on soil, AArslan 40.

133. Rhynchostegium megapolitanum (Blandow ex F.Weber \& D.Mohr) Schimp. - 13, on soil at forest road slope, AArslan 237.

134. Rhynchostegium riparioides (Hedw.) Cardot 8,13 , on wet rocks near stream, AArslan 85.

Hypnaceae Schimp.

135. Hypnum andoi A.J.E.Sm. - 1, 8, 19, on soil and deadwood, AArslan 153.

136. Hypnum cupressiforme Hedw. var. cupressiforme - 1, 2, 9, 21, on soil and tree root, AArslan 274.

137. Hypnum cupressiforme Hedw. var. lacunosum Brid. - 1, 8, 11, on soil and rocks, AArslan 111.

138. Hypnum jutlandicum Holmen \& E.Warncke - 17, on rocks, AArslan 443.

139. Hypnum resupinatum Taylor (Syn: $H$. cupressiforme var. resupinatum (Taylor) Schimp.) - 11, on bark, AArslan 195.

Pylaisiaceae Schimp.

140. Buckia vaucheri (Lesq.) D.Rios, M.T.Gallego \& J.Guerra (Syn: Hypnum vaucheri Lesq.) 11, on soil and rocks, AArslan 96.

141. Calliergonella cuspidata (Hedw.) Loeske - 8, 10, 22, on wet or damp soil, AArslan 177.
142. Homomallium incurvatum (Schrad. ex Brid.) Loeske - 17, on rocks, AArslan 244.

143. Pylaisia polyantha (Hedw.) Schimp. - 5, on barks, AArslan 115.

Hylocomiaceae M.Fleisch

144. Hylocomiadelphus triquetrus (Hedw.) Ochyra \& Stebel (Syn: Rhytidiadelphus triquetrus (Hedw.) Warnst.) - 8, on soil, AArslan 101.

145. Pleurozium schreberi (Willd. ex Brid.) Mitt. 8, 25, on soil, AArslan 167.

Rhytidiaceae Broth.

146. Rhytidium rugosum (Hedw.) Kindb. - 1, 4, 15, 25 , on soil at black pine forest, AArslan 9.

Leucodontaceae Schimp.

147. Leucodon sciuroides (Hedw.) Schwägr. var. morensis (Schwägr.) De Not. - 1, 8, 20, on barks, AArslan 129.

148. Leucodon sciuroides (Hedw.) Schwägr. var. sciuroides - 11, on barks, AArslan 8.

Antitrichiaceae Ignatov \& Ignatova

149. Antitrichia curtipendula (Hedw.) Brid. - 1, 9, on barks, AArslan 165.

Neckeraceae Schimp

150. Alleniella complanata (Hedw.) S.Olsson, Enroth \& D.Quandt (Syn. Neckera complanata (Hedw.) Huebener) - 19, 29, on barks, AArslan 39.

151. Exertotheca crispa (Hedw.) S.Olsson, Enroth \& D.Quandt (Syn. Neckera crispa Hedw.) 29, on barks, AArslan 53.

152. Pseudanomodon attenuatus (Hedw.) Ignatov \& Fedosov (Syn: Anomodon attenuatus (Hedw.) Huebener) - 29, on barks, AArslan 181.

153. Thamnobryum alopecurum (Hedw.) Gangulee - 10, on rocks, AArslan 112.

Lembophyllaceae Broth.

154. Isothecium alopecuroides (Lam. ex Dubois) Isov. - 2, on barks and roots, AArslan 272.

Myuriaceae M.Fleisch

155. Ctenidium molluscum (Hedw.) Mitt. - 6, 12, 19, on rocks and soil, AArslan 231.

Anomodontaceae Kindb.

156. Anomodon viticulosus (Hedw.) Hook. \& Taylor - 7, 13, on rocks, AArslan 145. 